\title{
Pseudo-Hermiticity versus PT-symmetry III: Equivalence of pseudo-Hermiticity and the presence of antilinear symmetries
}

Ali Mostafazadeh

Citation: Journal of Mathematical Physics 43, 3944 (2002); doi: 10.1063/1.1489072

View online: http://dx.doi.org/10.1063/1.1489072

View Table of Contents: http://aip.scitation.org/toc/jmp/43/8

Published by the American Institute of Physics

\section{Articles you may be interested in}

Pseudo-Hermiticity versus PT symmetry: The necessary condition for the reality of the spectrum of a non-

Hermitian Hamiltonian

Journal of Mathematical Physics 43, 205 (2001); 10.1063/1.1418246

Pseudo-Hermiticity versus PT-symmetry. II. A complete characterization of non-Hermitian Hamiltonians with a real spectrum

Journal of Mathematical Physics 43, 2814 (2016); 10.1063/1.1461427

Must a Hamiltonian be Hermitian?

American Journal of Physics 71, 1095 (2003); 10.1119/1.1574043

Pseudo-Hermiticity and generalized PT- and CPT-symmetries

Journal of Mathematical Physics 44, 974 (2003); 10.1063/1.1539304

PT-symmetric quantum mechanics

Journal of Mathematical Physics 40, 2201 (1999); 10.1063/1.532860

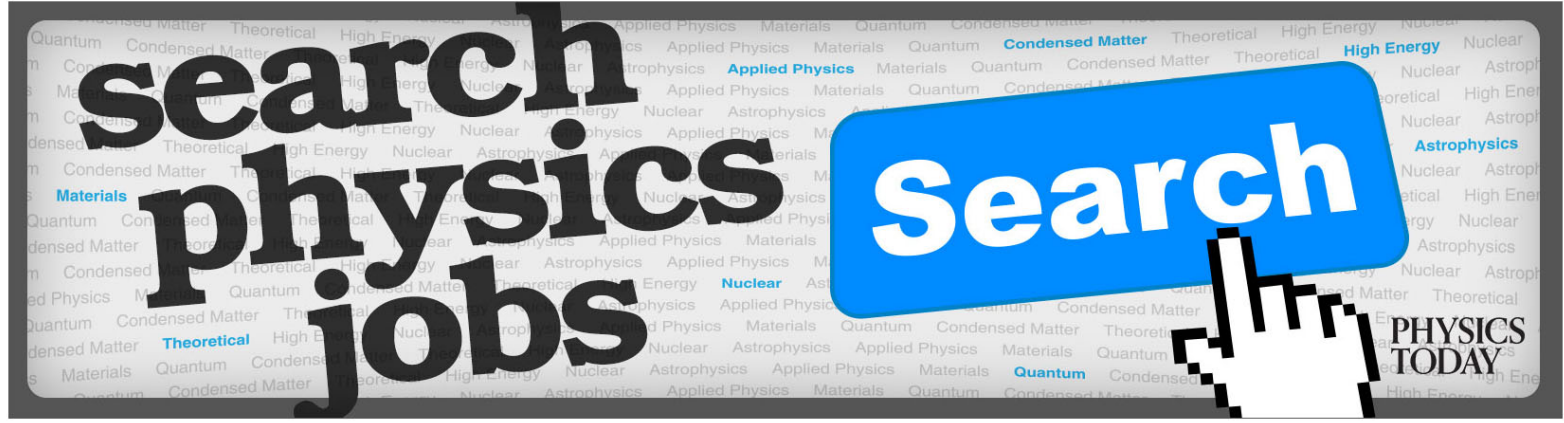




\title{
Pseudo-Hermiticity versus PT-symmetry III: Equivalence of pseudo-Hermiticity and the presence of antilinear symmetries
}

\author{
Ali Mostafazadeh ${ }^{\text {a) }}$ \\ Department of Mathematics, Koç University, Rumelifeneri Yolu, \\ 80910 Sariyer, Istanbul, Turkey
}

(Received 13 February 2002; accepted for publication 22 April 2002)

\begin{abstract}
We show that a diagonalizable (non-Hermitian) Hamiltonian $H$ is pseudoHermitian if and only if it has an antilinear symmetry, i.e., a symmetry generated by an invertible antilinear operator. This implies that the eigenvalues of $H$ are real or come in complex conjugate pairs if and only if $H$ possesses such a symmetry. In particular, the reality of the spectrum of $H$ implies the presence of an antilinear symmetry. We further show that the spectrum of $H$ is real if and only if there is a positive-definite inner-product on the Hilbert space with respect to which $H$ is Hermitian or alternatively there is a pseudo-canonical transformation of the Hilbert space that maps $H$ into a Hermitian operator. (C) 2002 American Institute of Physics. [DOI: 10.1063/1.1489072]
\end{abstract}

\section{INTRODUCTION}

The main reason for the recent interest in $P T$-symmetry ${ }^{1}$ is that the eigenvalues of every $P T$-symmetric Hamiltonian are real or come in complex conjugate pairs. In particular, if the $P T$-symmetry is exact, the spectrum of the Hamiltonian is real. In Ref. 2, we introduced the concept of a pseudo-Hermitian operator and showed that the remarkable spectral properties of the PT-symmetric Hamiltonians follow from their pseudo-Hermiticity. Under the assumption of the diagonalizability (equivalently the existence of a complete biorthonormal set of energy eigenvectors), we obtained in Ref. 3 a complete characterization of all the (non-Hermitian) Hamiltonians that have a real spectrum. Here we also pointed out that the spectral properties of the $P T$-symmetric Hamiltonians are common to all Hamiltonians possessing an antilinear symmetry (a symmetry generated by an invertible antilinear operator). Therefore, at least for the class of diagonalizable Hamiltonians, presence of an antilinear symmetry implies pseudo-Hermiticity of the Hamiltonian. The main purpose of the present article is to show that the converse of this statement holds as well, that is, pseudo-Hermiticity of a Hamiltonian implies the existence of an antilinear symmetry. A direct consequence of this result is that if the spectrum of the Hamiltonian is real, then the system has an antilinear symmetry, $P T$-symmetry being the prime example.

The organization of the article is as follows. Section II includes a brief review of the necessary results reported in the companion articles. ${ }^{2,3}$ Section III examines anti-pseudo-Hermiticity (pseudo-Hermiticity with an antilinear automorphism.) Here we prove that every (non-Hermitian) diagonalizable Hamiltonian is anti-pseudo-Hermitian and that the pseudo-Hermiticity of the Hamiltonian implies the presence of an antilinear symmetry. Section IV offers a description of the Hamiltonians with a real spectrum in terms of certain associated Hermitian operators. Section V presents a summary of the main results and the concluding remarks.

Throughout this article we shall consider (non-Hermitian) Hamiltonians $H$ that are diagonalizable and have a discrete spectrum. As we explain below, this means that these Hamiltonians admit a complete biorthonormal set of eigenvectors $\left\{\left(\left|\psi_{n}, a\right\rangle,\left|\phi_{n}, a\right\rangle\right)\right\}$. The latter satisfy the following defining relations: ${ }^{4}$

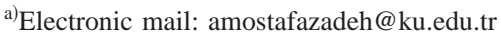




$$
\begin{aligned}
H\left|\psi_{n}, a\right\rangle= & E_{n}\left|\psi_{n}, a\right\rangle, \quad H^{\dagger}\left|\phi_{n}, a\right\rangle=E_{n}^{*}\left|\phi_{n}, a\right\rangle, \\
& \left\langle\phi_{m}, b \mid \psi_{n}, a\right\rangle=\delta_{m n} \delta_{a b}, \\
& \sum_{n} \sum_{a=1}^{d_{n}}\left|\psi_{n}, a\right\rangle\left\langle\phi_{n}, a\right|=1,
\end{aligned}
$$

where $n$ and $a$ are, respectively, the spectral and degeneracy labels, $d_{n}$ is the multiplicity (degree of degeneracy) of $E_{n}$, $\dagger$ and $*$, respectively, denote the adjoint and complex-conjugate, $\delta_{m n}$ stands for the Kronecker delta function, and 1 is the identity operator. In view of Eqs. (1)-(3), we also have

$$
H=\sum_{n} \sum_{a=1}^{d_{n}} E_{n}\left|\psi_{n}, a\right\rangle\left\langle\phi_{n}, a\left|, \quad H^{\dagger}=\sum_{n} \sum_{a=1}^{d_{n}} E_{n}^{*}\right| \phi_{n}, a\right\rangle\left\langle\psi_{n}, a\right| .
$$

In order to see the equivalence of the existence of a complete biorthonormal set of eigenvectors of $H$ and its diagonalizability, we note that by definition a diagonalizable Hamiltonian $H$ satisfies $A^{-1} H A=H_{0}$ for an invertible linear operator $A$ and a diagonal linear operator $H_{0}$, i.e., there is an orthonormal basis $\{|n, \alpha\rangle\}$ in the Hilbert space and complex numbers $E_{n}$ such that $H_{0}=\Sigma_{n} \Sigma_{\alpha} E_{n}|n, \alpha\rangle\langle n, \alpha|$. Then letting $\left|\psi_{n}, \alpha\right\rangle:=A|n, \alpha\rangle$ and $\left|\phi_{n}, \alpha\right\rangle:=\left(A^{-1}\right)^{\dagger}|n, \alpha\rangle$, we can easily check that $\left\{\left|\psi_{n}, \alpha\right\rangle,\left|\psi_{n}, \alpha\right\rangle\right\}$ is a complete biorthonormal system for $H$. The converse is also true, for if such a system exists we may set $A:=\Sigma_{n} \Sigma_{\alpha}\left|\psi_{n}, \alpha\right\rangle\langle n, \alpha|$ for some orthonormal basis $\{|n, \alpha\rangle\}$ and check that $A^{-1}=\Sigma_{n} \Sigma_{\alpha}|n, \alpha\rangle\left\langle\phi_{n}, \alpha\right|$ and $A^{-1} H A=H_{0}$, i.e., $H$ is diagonalizable.

We would like to emphasize that the diagonalizability condition may be viewed as a physical requirement without which an energy eigenbasis would not exist. To our knowledge all known non-Hermitian Hamiltonians that are used in physical applications are diagonalizable and therefore admit a complete biorthonormal set of eigenvectors. This in particular includes all the Hermitian Hamiltonians as well as the non-Hermitian Hamiltonians used in ionization optics, ${ }^{5}$ the study of dissipative systems and resonant states, ${ }^{6}$ two-component formulation of the minisuperspace quantum cosmology, ${ }^{7}$ and also the $P T$-symmetric Hamiltonians whose spectral properties have been obtained using numerical methods.

\section{PSEUDO-HERMITICITY}

Let $H: \mathcal{H} \rightarrow \mathcal{H}$ be a linear operator acting in a Hilbert space $\mathcal{H}$ and $\eta: \mathcal{H} \rightarrow \mathcal{H}$ be a linear Hermitian automorphism (invertible transformation). Then the $\eta$-pseudo-Hermitian adjoint of $H$ is defined $b y^{2}$

$$
H^{\sharp}:=\eta^{-1} H^{\dagger} \eta
$$

$H$ is said to be pseudo-Hermitian with respect to $\eta$ or simply $\eta$-pseudo-Hermitian if $H^{\sharp}=H$. $H$ is said to be pseudo-Hermitian if it is pseudo-Hermitian with respect to some linear Hermitian automorphism $\eta$.

The basic properties of pseudo-Hermitian operators are discussed in Refs. 2 and 3. Here we survey the properties that we shall make use of in this article. Let $H: \mathcal{H} \rightarrow \mathcal{H}$ be a diagonalizable linear operator. Then

(i) $\quad H$ is pseudo-Hermitian if and only if its eigenvalues are real or come in complex-conjugate pairs; ${ }^{2}$ and

(ii) if $H$ is pseudo-Hermitian with respect to two linear Hermitian automorphisms $\eta_{1}$ and $\eta_{2}$, then $\eta_{1}^{-1} \eta_{2}$ generates a symmetry of $H$, i.e., $\left[H, \eta_{1}^{-1} \eta_{2}\right]=0 .^{2}$ 


\section{ANTI-PSEUDO-HERMITICITY}

We first recall that a function $\tau: \mathcal{H} \rightarrow \mathcal{H}$ acting in a (complex) Hilbert space $\mathcal{H}$ is said to be an antilinear operator if for all $a, b \in \mathrm{C}$ and $|\xi\rangle,|\zeta\rangle \in \mathcal{H}$,

$$
\tau(a|\xi\rangle+b|\zeta\rangle)=a^{*} \tau|\xi\rangle+b^{*} \tau|\zeta\rangle .
$$

An antilinear operator $\tau: \mathcal{H} \rightarrow \mathcal{H}$ is said to be anti-Hermitian ${ }^{8}$ if for all $|\xi\rangle,|\zeta\rangle \in \mathcal{H}$,

$$
\langle\zeta|\tau| \xi\rangle=\langle\xi|\tau| \zeta\rangle .
$$

Definition 1: A linear operator $H: \mathcal{H} \rightarrow \mathcal{H}$ acting in a Hilbert space $\mathcal{H}$ is said to be anti-pseudoHermitian if there is an antilinear anti-Hermitian automorphism $\tau: \mathcal{H} \rightarrow \mathcal{H}$ satisfying

$$
H^{\dagger}=\tau H \tau^{-1} .
$$

We begin our analysis by giving a characterization of antilinear anti-Hermitian operators with respect to which a given linear operator is anti-pseudo-Hermitian.

Theorem 1: Let $\mathcal{H}$ be a Hilbert space and $H: \mathcal{H} \rightarrow \mathcal{H}$ be a diagonalizable linear operator with a discrete spectrum and a complete biorthonormal set of eigenvectors $\left\{\left(\left|\psi_{n}, a\right\rangle,\left|\phi_{n}, a\right\rangle\right)\right\}$. Then $\tau: \mathcal{H} \rightarrow \mathcal{H}$ is an antilinear anti-Hermitian operator and $H$ is $\tau$-anti-pseudo-Hermitian if and only if there are symmetric invertible matrices $c^{(n)}$ with entries $c_{a b}^{(n)}$ such that for all $|\zeta\rangle \in \mathcal{H}$,

$$
\tau|\zeta\rangle=\sum_{n} \sum_{a, b=1}^{d_{n}} c_{a b}^{(n)}\left\langle\zeta \mid \phi_{n}, a\right\rangle\left|\phi_{n}, b\right\rangle
$$

Proof: Suppose that $\tau: \mathcal{H} \rightarrow \mathcal{H}$ is a given antilinear anti-Hermitian operator and $H$ is $\tau$-antipseudo-Hermitian, i.e., (8) or equivalently

$$
H^{\dagger} \tau=\tau H
$$

holds. Letting both sides of (10) act on $\left|\psi_{n}, a\right\rangle$ and using (1) and (6), we have

$$
H^{\dagger}\left(\tau\left|\psi_{n}, a\right\rangle\right)=E_{n}^{*}\left(\tau\left|\psi_{n}, a\right\rangle\right)
$$

Comparing this equation with the second equation in (1), we find

$$
\tau\left|\psi_{n}, a\right\rangle=\sum_{b=1}^{d_{n}} c_{b a}^{(n)}\left|\phi_{n}, b\right\rangle
$$

where $c_{a b}^{(n)}$ are defined by

$$
c_{a b}^{(n)}:=\left\langle\psi_{n, a}|\tau| \psi_{n}, a\right\rangle .
$$

We can also express (11) in the form

$$
\left\langle\psi_{m}, b|\tau| \psi_{n}, a\right\rangle=\delta_{m n} c_{b a}^{(n)} .
$$

Next note that because $\tau$ is an invertible operator, the matrix $c^{(n)}=\left(c_{a b}^{(n)}\right)$ formed by $c_{a b}^{(n)}$ is nonsingular. In fact, applying $\left\langle\phi_{n, c}\right| \tau^{-1}$ to both sides of (11) and using (6) and the fact that $\tau^{-1}$ is also antilinear, we have

$$
\left(c^{(n)^{-1}}\right)_{a b}=\left\langle\phi_{n}, a\left|\tau^{-1}\right| \phi_{n}, b\right\rangle^{*} .
$$

Furthermore, in view of (12) and (7), $c^{(n)}$ is a symmetric matrix. Now let $|\zeta\rangle$ be an arbitrary element of $\mathcal{H}$ and use (13), (3) and (7) to compute 


$$
\begin{aligned}
\sum_{n} \sum_{a, b=1}^{d_{n}} c_{a b}^{(n)}\left\langle\zeta \mid \phi_{n}, a\right\rangle\left|\phi_{n}, b\right\rangle & =\sum_{n, m} \sum_{a, b=1}^{d_{n}}\left|\phi_{m}, b\right\rangle\left\langle\zeta \mid \phi_{n}, a\right\rangle\left\langle\psi_{n}, a|\tau| \psi_{m}, b\right\rangle \\
& =\sum_{m} \sum_{b}\left|\phi_{m}, b\right\rangle\left\langle\zeta|\tau| \psi_{m}, b\right\rangle \\
& =\sum_{m} \sum_{b}\left|\phi_{m}, b\right\rangle\left\langle\psi_{m}, b|\tau| \zeta\right\rangle \\
& =\tau|\zeta\rangle .
\end{aligned}
$$

This establishes (9). Next, suppose that $c^{(n)}$ are given invertible symmetric matrices and $\tau$ is defined by (9). Then the antilinearity of $\tau$ follows from the antilinearity of the inner-product in its first entry. The following simple calculation shows that $\tau$ is anti-Hermitian. For all $|\xi\rangle,|\zeta\rangle \in \mathcal{H}$,

$$
\begin{aligned}
\langle\xi|\tau| \zeta\rangle & =\sum_{n} \sum_{a, b=1}^{d_{n}} c_{a b}^{(n)}\left\langle\zeta \mid \phi_{n}, a\right\rangle\left\langle\xi \mid \phi_{n}, b\right\rangle \\
& =\sum_{n} \sum_{a, b=1}^{d_{n}} c_{a b}^{(n)}\left\langle\zeta \mid \phi_{n}, b\right\rangle\left\langle\xi \mid \phi_{n}, a\right\rangle \\
& =\sum_{n} \sum_{a, b=1}^{d_{n}} c_{a b}^{(n)}\left\langle\xi \mid \phi_{n}, a\right\rangle\left\langle\zeta \mid \phi_{n}, b\right\rangle \\
& =\langle\zeta|\tau| \xi\rangle,
\end{aligned}
$$

where we used (9) and the fact that $c^{(n)}$ are symmetric. In order to establish the $\tau$-anti-pseudoHermiticity of $H$ we first observe that (9) implies

$$
\tau^{-1}|\zeta\rangle=\sum_{n} \sum_{a, b=1}^{d_{n}}\left(c^{(n)}\right)_{a b}^{-1 *}\left\langle\zeta \mid \psi_{n}, a\right\rangle\left|\psi_{n}, b\right\rangle
$$

This can be easily checked by applying $\tau$ to the right-hand side of (15) and using (9), (2), and (3) to show that the result is $|\zeta\rangle$. Next, we note that applying both sides of (9) to $\left|\psi_{n}, a\right\rangle$ we recover (11). Finally, we use (9), (15), (6), (1), (4), and (11) to compute, for all $|\zeta\rangle \in \mathcal{H}$,

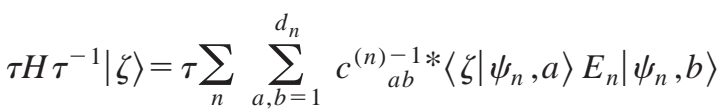

$$
\begin{aligned}
& =\sum_{n} \sum_{a, b=1}^{d_{n}} E_{n}^{*} c_{a b}^{(n)-1}\left\langle\zeta \mid \psi_{n}, a\right\rangle^{*} \tau\left|\psi_{n}, b\right\rangle \\
& =\sum_{n} \sum_{a, b, c=1}^{d_{n}} E_{n}^{*}\left\langle\psi_{n}, a \mid \zeta\right\rangle c_{c a}^{(n)} c_{a b}^{(n)-1}\left|\phi_{n}, c\right\rangle \\
& =\sum_{n} \sum_{b=1}^{d_{n}} E_{n}^{*}\left|\phi_{n}, b\right\rangle\left\langle\psi_{n}, b \mid \zeta\right\rangle=H^{\dagger}|\zeta\rangle .
\end{aligned}
$$

Therefore, $\tau H \tau^{-1}=H^{\dagger}$.

We should emphasize that, unlike the case of pseudo-Hermitian Hamiltonians, the antipseudo-Hermiticity does not restrict the energy spectrum. In fact, we can use Theorem 1 to prove the following. 
Corollary 1: Every diagonalazable linear operator $H: \mathcal{H} \rightarrow \mathcal{H}$ with a discrete spectrum is antipseudo-Hermitian.

Proof: Let $\left\{\left(\left|\psi_{n}, a\right\rangle,\left|\phi_{n}, a\right\rangle\right)\right\}$ be a complete biorthonormal set of eigenvectors, and $\tau: \mathcal{H}$ $\rightarrow \mathcal{H}$ be defined by (9) with $c^{(n)}=1$ for all $n$, i.e., for all $|\zeta\rangle \in \mathcal{H}$,

$$
\tau|\zeta\rangle:=\sum_{n} \sum_{a=1}^{d_{n}}\left\langle\zeta \mid \phi_{n}, a\right\rangle\left|\phi_{n}, a\right\rangle .
$$

Then according to Theorem 1, $\tau$ is an antilinear anti-Hermitian operator and $H$ is $\tau$-anti-pseudoHermitian.

Corollary 2: Every diagonalizable pseudo-Hermitian linear operator $H: \mathcal{H} \rightarrow \mathcal{H}$ with a discrete spectrum has an antilinear symmetry.

Proof: Let $H$ be pseudo-Hermitian. Then according to Corollary 1 it is also anti-pseudoHermitian, i.e., there are a linear Hermitian automorphism $\eta: \mathcal{H} \rightarrow \mathcal{H}$ and an antilinear antiHermitian automorphism $\tau: \mathcal{H} \rightarrow \mathcal{H}$ such that

$$
\eta H \eta^{-1}=H^{\dagger}=\tau H \tau^{-1} .
$$

Hence, $\left[H, \eta^{-1} \tau\right]=0$. Clearly $\eta^{-1} \tau$ is an antilinear operator.

Theorem 2: Let $H: \mathcal{H} \rightarrow \mathcal{H}$ be a diagonalizable linear operator acting in a Hilbert space $\mathcal{H}$ with a discrete spectrum. Then the following are equivalent.

(1) The eigenvalues of $H$ are real or come in complex-conjugate pairs.

(2) $H$ is pseudo-Hermitian.

(3) $H$ has an antilinear symmetry.

Proof: The equivalence of (1) and (2) was established in Ref. 2; Corollary 2 shows that (2) implies (3); the fact that (3) implies (1) follows from a simple calculation given in Ref. 3.

A class of $P T$-symmetric Hamiltonians is given by

$$
H=\frac{p^{2}}{2 m}+V_{1}(x)+i V_{2}(x),
$$

where $V_{1}$ and $V_{2}$ are, respectively, even and odd real-valued functions and the classical phase space is assumed to be real, i.e., $x$ and $p$ are the standard Hermitian operators representing the position and momentum of a particle of mass $m$. As we point out in Ref. 2, the Hamiltonian (18) is $P$-pseudo-Hermitian. It is also easy to check that it is $T$-anti-pseudo-Hermitian. The $P$-pseudo-Hermiticity and $T$-anti-pseudo-Hermiticity of this Hamiltonian implies its $P^{-1} T=P T$ symmetry. In general, there are $P T$-symmetric Hamiltonians $H$ that are neither $P$-pseudo-Hermitian nor $T$-anti-pseudo-Hermitian. According to Theorem 2, if we make the physical assumption that $H$ is diagonalizable, so that it admits a complete biorthonormal set of energy eigenvectors, then $H$ must be pseudo-Hermitian with respect to a linear Hermitian automorphism $\eta$. It turns out that the choice of $\eta$ is not unique. But fixing an antilinear anti-Hermitian operator $\tau$ with respect to which $H$ is anti-pseudo-Hermitian [namely, (9)], we can express $\eta$ in terms of $P T$ and $\tau$ according to

$$
\eta=\tau P T .
$$

One can easily check that $P T$-symmetry $([P T, H]=0)$ and anti-pseudo-Hermiticity (8) imply pseudo-Hermiticity of $H$ with respect to (19).

Next we consider a general diagonalizable Hamiltonian $H$ with a discrete spectrum and a symmetry generated by a general invertible antilinear operator $X$,

$$
[H, X]=0 .
$$


The antilinearity of $X$ implies $\eta$-pseudo-Hermiticity of $H$ with respect to some linear Hermitian automorphism $\eta$. The anti-pseudo-Hermiticity of $H$ with respect to an antilinear automorphism of the form (9) always holds. Hence Eqs. (17) are valid. Taking the adjoint of both sides of (20) and making use of (17), we can easily show that $X_{\eta}^{\sharp}:=\eta^{-1} X \eta$ and $X_{\tau}^{\sharp}:=\tau^{-1} X \tau$ commute with $H$, i.e., they generate antilinear symmetries of the system as well.

\section{NON-HERMITIAN HAMILTONIANS WITH A REAL SPECTRUM}

We first recall the following results which we reported in Refs. 2 and 3.

(1) The (indefinite) inner-product defined by

$$
\forall|\xi\rangle,|\zeta\rangle \in \mathcal{H}, \quad\langle\langle\xi \mid \zeta\rangle\rangle:=\langle\xi|\eta| \zeta\rangle,
$$

is invariant under the evolution generated by an $\eta$-pseudo-Hermitian Hamiltonian $H .^{2}$ It is also easy to check that such a Hamiltonian is Hermitian with respect to the (indefinite) inner-product (21). See also Ref. 9.

(2) A diagonalizable (non-Hermitian) Hamiltonian has a real spectrum if and only if it is pseudo-Hermitian with respect to a linear Hermitian automorphism of the form

$$
\eta=O O^{\dagger},
$$

where $O: \mathcal{H} \rightarrow \mathcal{H}$ is a linear automorphism. ${ }^{3}$

These statements suggest the following characterization of the (non-Hermitian) Hamiltonians with a real spectrum. See also Ref. 10.

Theorem 3: A diagonalizable Hamiltonian $H$ acting in a Hilbert space $\mathcal{H}$ has a real spectrum if and only if there is a positive-definite inner-product on $\mathcal{H}$ with respect to which $H$ is Hermitian.

Proof: Suppose $H$ has a real spectrum so that it is $O O^{\dagger}$-pseudo-Hermitian for a linear automorphism $O: \mathcal{H} \rightarrow \mathcal{H}$. Then the inner-product (21) with $\eta=O O^{\dagger}$ is clearly a positive-definite inner-product with respect to which $H$ is Hermitian. Conversely, suppose that there is a positivedefinite inner-product $($,$) with respect to which H$ is Hermitian. Then treating the spectral problem for $H$ in the Hilbert space $\mathcal{H}$ with the inner-product (, ), we find that $H$ has a real spectrum.

Corollary: Suppose that $H$ has an antilinear symmetry $X$. If $X$ is an exact symmetry of $H$, then there is a positive-definite inner product on $\mathcal{H}$ with respect to which $H$ is Hermitian.

Proof: Exactness of an antilinear symmetry implies reality of the spectrum of $H^{3}$ The conclusion then follows from Theorem 3.

Next we give an alternative and in a sense equivalent characterization of the (non-Hermitian) Hamiltonians with a real spectrum.

Definition 2: Consider a quantum system with the Hilbert space $\mathcal{H}$ and the Hamiltonian $H: \mathcal{H} \rightarrow \mathcal{H}$. Then a linear automorphism $A: \mathcal{H} \rightarrow \mathcal{H}$ is said to be a pseudo-canonical transformation for the system if for all $|\zeta\rangle \in \mathcal{H}$ the transformation

$$
|\zeta\rangle \rightarrow|\widetilde{\zeta}\rangle:=A|\zeta\rangle
$$

leaves the Schrödinger equation,

$$
i \frac{d}{d t}|\psi(t)\rangle=H|\psi(t)\rangle
$$

form-invariant. A unitary pseudo-canonical transformation is called a (quantum) canonical transformation. ${ }^{11}$

Clearly the defining condition for a pseudo-canonical transformation implies the following transformation rule for the Hamiltonian:

$$
H \rightarrow \widetilde{H}:=A H A^{-1}+i \dot{A} A^{-1},
$$


where a dot denotes a time-derivative. For a time-independent pseudo-canonical transformation $A$, the second term on the right-hand side of (25) drops and $H$ transforms as

$$
H \rightarrow \widetilde{H}:=A H A^{-1} .
$$

Theorem 4: A diagonalizable time-independent Hamiltonian $H$ has a real spectrum if and only if there is a pseudo-canonical transformation that maps $H$ into a Hermitian operator.

Proof: Suppose that $H$ has a real spectrum. Then it is $O O^{\dagger}$-pseudo-Hermitian for a linear automorphism $O: \mathcal{H} \rightarrow \mathcal{H}$, i.e., $H^{\dagger}=O O^{\dagger} H\left(O O^{\dagger}\right)^{-1}$. Let $A:=O^{\dagger}$. Then, in view of (26) and the preceding equation, we have

$$
\widetilde{H}^{\dagger}=\left(A H A^{-1}\right)^{\dagger}=\left(A^{-1}\right)^{\dagger} H^{\dagger} A^{\dagger}=\left(A^{-1}\right)^{\dagger} A^{\dagger} A H A^{-1}\left(A^{\dagger}\right)^{-1} A^{\dagger}=A H A^{-1}=\widetilde{H} .
$$

Hence the transformed Hamiltonian is Hermitian. Conversely suppose that there is a pseudocanonical transformation $A: \mathcal{H} \rightarrow \mathcal{H}$ under which $H$ transforms to a Hermitian Hamiltonian $\widetilde{H}$ and let $O:=A^{\dagger}$. Then using (26) and $\widetilde{H}^{\dagger}=\widetilde{H}$, we have

$$
O O^{\dagger} H\left(O O^{\dagger}\right)^{-1}=A^{\dagger} A A^{-1} \widetilde{H} A\left(A^{\dagger} A\right)^{-1}=A^{\dagger} \widetilde{H}^{\dagger}\left(A^{\dagger}\right)^{-1}=\left(A^{-1} \widetilde{H} A\right)^{\dagger}=H^{\dagger} .
$$

Therefore, $H$ is $O O^{\dagger}$-pseudo-Hermitian, and its spectrum is real.

Corollary: Suppose that $H$ has an antilinear symmetry $X$. If $X$ is an exact symmetry of $H$, then there is a pseudo-canonical transformation that maps $H$ into a Hermitian operator.

Proof: Exactness of an antilinear symmetry implies reality of the spectrum of $H{ }^{3}$ The conclusion then follows from Theorem 4.

\section{DISCUSSION AND CONCLUSION}

In this article we established the equivalence of the notion of pseudo-Hermiticity and presence of an antilinear symmetry for the class of diagonalizable (non-Hermitian) Hamiltonians. This required the study of pseudo-Hermiticity with respect to antilinear anti-Hermitian automorphisms. It turned that the latter does not restrict the choice of the Hamiltonian and such antilinear automorphisms always exist. In fact, we obtained the general form of these automorphisms. For a fixed complete biorthonormal eigenbasis, they are determined in terms of a sequence of complex symmetric matrices $c^{(n)}$. The choice of unity for all these matrices leads to a canonical antilinear anti-Hermitian automorphism, namely (16). Under an invertible transformation $u$ of the basis

$$
\left|\psi_{n}, a\right\rangle \rightarrow \sum_{b=1}^{d_{n}} u_{b a}\left|\psi_{n}, b\right\rangle, \quad\left|\phi_{n}, a\right\rangle \rightarrow \sum_{b=1}^{d_{n}}\left(u^{-1 \dagger}\right)_{b a}\left|\phi_{n}, b\right\rangle
$$

that preserves its completeness and biorthonormality, the matrices $c^{(n)}$ transform according to

$$
c^{(n)} \rightarrow u^{* t} c^{(n)} u^{*}=u^{\dagger} c^{(n)} u^{\dagger t},
$$

where ${ }^{t}$ denotes the transpose. We can transform to a basis where a general $\tau$ has the canonical form (16) if we can find invertible matrices $v=u^{-1 \dagger}$ satisfying $c^{(n)}=v v^{t}$. As shown in Ref. 12 this is always possible. Therefore, up to the choice of the biorthonormal eigenbasis, $\tau$ is actually unique.

A simple consequence of our findings is that the reality of the spectrum of a Hamiltonian implies the presence of an antilinear symmetry. In view of the proof of Corollary 2 and Eq. (9) of this article and Eq. (23) of Ref. 2, we have in fact an explicit expression for the generator of such a symmetry in terms of the biorthonormal eigenvectors of the Hamiltonian. We also gave two characterizations of Hamiltonians with real spectrum. These characterizations show how a Hamiltonian with a real spectrum may be related to an associated Hermitian Hamiltonian. Another simple implication of our analysis is that every Hermitian Hamiltonian has an antilinear symmetry. 


\section{ACKNOWLEDGMENT}

This project was supported by the Young Researcher Award Program (GEBIP) of the Turkish Academy of Sciences.

${ }^{1}$ C. M. Bender and S. Boettcher, Phys. Rev. Lett. 80, 5243 (1998); F. M. Fernández, R. Guardiola, J. Ros, and M. Znojil, J. Phys. A 31, 10105 (1998); F. Cannata, G. Junker, and J. Trost, Phys. Lett. A 246, 219 (1998); C. M. Bender, S. Boettcher, and P. N. Meisenger, J. Math. Phys. 40, 2201 (1999); C. M. Bender, G. V. Dunne, and P. N. Meisenger, Phys. Lett. A 252, 272 (1999); C. M. Bender and G. V. Dunne, J. Math. Phys. 40, 4616 (1999); G. A. Mezincescu, J. Phys. A 33, 4911 (2000); E. Delabaere and D. T. Trinh, ibid. 33, 8771 (2000); B. Bagchi and R. Roychoudhury, ibid. 33, L1 (2000); A. Khare and B. P. Mandal, Phys. Lett. A 272, 53 (2000); B. Bagchi, F. Cannata, and C. Quesne, ibid. 269, 79 (2000); M. Znojil, F. Cannata, B. Bagchi, and R. Roychoudhury, Phys. Lett. B 483, 284 (2000); M. Znojil and M. Tater, J. Phys. A 34, 1793 (2001); C. M. Bender, G. V. Dunne, P. N. Meisenger, and M. Şimşek, Phys. Lett. A 281, 311 (2001); F. Cannata, M. Ioffe, R. Roychoudhury, and P. Roy, ibid. 281, 305 (2001); Z. Ahmed, ibid. 282, 343 (2001); 284, 231 (2001); 290, 19 (2001); P. Dorey, C. Dunning, and R. Tateo, J. Phys. A 34, 5679 (2001); K. C. Shin, J. Math. Phys. 42, 2513 (2001); "On the reality of the eigenvalues for a class of $P T$-symmetric oscillators," LANL archives, math-ph/0201013. See also S. M. Klishevich and M. Plyushchay, Nucl. Phys. B 628, 217 (2002).

${ }^{2}$ A. Mostafazadeh, J. Math. Phys. 43, 205 (2002).

${ }^{3}$ A. Mostafazadeh, J. Math. Phys. 43, 2814 (2002)

${ }^{4}$ J. Wong, J. Math. Phys. 8, 2039 (1967).

${ }^{5}$ F. H. M. Faisal and J. V. Moloney, J. Phys. B 14, 3603 (1981); H. Baker, Phys. Rev. Lett. 50, 1579 (1983); Phys. Rev. A 30, 773 (1984); G. Dattoli, R. Mignani, and A. Torre, J. Phys. A 23, 5795 (1990); G. Dattoli, A. Torre, and R. Mignani, Phys. Rev. A 42, 1467 (1990).

${ }^{6}$ J. C. Garrison and E. M. Wright, Phys. Lett. A 128, 177 (1988); A. Kvitsinsky and S. Putterman, J. Math. Phys. 32, 1403 (1991); A. Mondragón and E. Hernández, J. Phys. A 29, 2567 (1996).

${ }^{7}$ A. Mostafazadeh, J. Math. Phys. 39, 4499 (1998).

${ }^{8}$ S. Weinberg, The Quantum Theory of Fields (Cambridge U. P., Cambridge, 1995), Vol. I.

${ }^{9}$ G. S. Japaridze, J. Phys. A 35, 1709 (2002).

${ }^{10}$ R. Kretschmer and L. Szymanowski, "The interpretation of quantum-mechanical models with non-Hermitian Hamiltonians and real spectra," LANL Archives, quant-ph/0105054.

${ }^{11}$ A. Mostafazadeh, Dynamical Invariants, Adiabatic Approximation, and the Geometric Phase (Nova Science, New York, 2001).

${ }^{12}$ A. Mostafazadeh, "On a Factorization of Symmetric Matrices and Antilinear Symmetries," LANL Archives, math-ph/0203023. 